\title{
Spectral Methods with Postprocessing for Numerical Hyperbolic Heat Transfer
}

\author{
Scott A. Sarra \\ Department of Mathematics, Marshall University, One John Marshall Drive, \\ Huntington, $W V, 25755-2560$.
}

\begin{abstract}
Under the governing equations of Hyperbolic Heat Transfer, energy propagates through a medium as a wave with sharp discontinuities at the wave front. The use of spectral methods to solve such problems numerically results in a solution in which strong numerical oscillations are present due to the Gibbs-Wilbraham Phenomenon. It is demonstrated that a spectrally accurate solution can still be obtained via a postprocessing technique.
\end{abstract}

NOMENCLATURE

Email address: scott@scottsarra.org (Scott A. Sarra).

$U R L$ : www.scottsarra.org (Scott A. Sarra). 


$$
\begin{aligned}
& a_{n} \quad \text { discrete Chebyshev coefficients } \\
& a_{n}^{(1)} \quad \text { discrete Chebyshev coefficients, } 1^{\text {st }} \text { derivative } \\
& C_{n}^{\lambda} \quad n^{\text {th }} \text { order Gegenbauer polynomial } \\
& \widehat{f}_{l}^{\lambda} \quad \text { continuous Gegenbauer coefficients } \\
& {[f](x) \text { function discontinuity }} \\
& \widehat{g}_{l}^{\lambda} \quad \text { approximate Gegenbauer coefficients } \\
& \widehat{g}_{\epsilon}^{\lambda}(l) \quad \text { approximate Gegenbauer coefficients in a subinterval } \\
& h_{n}^{\lambda} \quad \text { Gegenbauer normalization function } \\
& \text { J edge detection critical threshold } \\
& k_{m} \quad \text { global Gegenbauer reconstruction parameter } \\
& k_{\lambda} \quad \text { global Gegenbauer reconstruction parameter } \\
& \mathrm{S}(\mathrm{x}, \mathrm{t}) \text { dimensionless energy generation rate } \\
& \mathrm{T}(\mathrm{x}, \mathrm{t}) \text { dimensionless temperature } \\
& T_{k} \quad k^{t h} \text { order Chebyshev polynomial } \\
& \mathrm{t} \text { dimensionless time } \\
& u_{N} \quad \text { Chebyshev partial sum } \\
& \text { ue }(x) \quad \text { edge series } \\
& \text { un }(x) \text { enhanced edge series } \\
& u_{m}^{\lambda, \epsilon} \quad \text { Gegenbauer approximation in a subinterval } \\
& \mathrm{Q}(\mathrm{x}, \mathrm{t}) \text { dimensionless heat flux } \\
& \text { Q edge detection enhancement exponent } \\
& \mathrm{x} \text { dimensionless space variable in }[a, b] \\
& \gamma \quad \text { coordinate map parameter } \\
& \Gamma \quad \text { Gamma function } \\
& \Delta t \quad \text { time step } \\
& \epsilon \quad \text { half the width of a subinterval }[a, b] \\
& \eta \quad \text { edge detection neighborhood parameter } \\
& \lambda \quad \text { Gegenbauer polynomial parameter } \\
& \psi \quad \text { dimensionless space variable in }[-1,1]
\end{aligned}
$$




\section{Introduction}

In situations when the elapsed time during a transient is very small or when temperatures near absolute zero are involved, the classical diffusion (parabolic) theory of heat transfer breaks down since the wave nature of thermal energy transport becomes dominant. The hyperbolic heat equation models this process and results in energy propagating through a medium as a wave with sharp discontinuities at the wave front.

The dimensionless governing equations of Hyperbolic Heat Transfer are

$$
\begin{aligned}
& T_{t}+Q_{x}=S / 2 \\
& Q_{t}+T_{x}=-2 Q
\end{aligned}
$$

where $T(x, t)$ is the temperature, $Q(x, t)$ is the heat flux, and $S(x, t)$ is the energy generation rate.

Previously in the literature, the numerical solutions of hyperbolic heat transfer problems have been obtained using the Finite Element Method in [1], and by MacCormack's method in [2], and [3]. In previous numerical investigations, typically 1000 grids points were used and numerical oscillations still remained in the solution. Non-oscillatory finite difference methods $[4,5]$ which suppress oscillation by using a flux or slope limiter are available. However, the methods are also know to smear the solution at sharp fronts. The spectral method with postprocessing will resolve all fronts sharply.

The first attempt to apply spectral methods to the problem was in [6] where conservative smoothing was used to obtain results with significantly fewer grid points than MacCormack's method while eliminating most of the spurious oscillations. Conservative smoothing amounts to an artificial viscosity, which can be applied selectively in both space and time. However, the global nature of spectral methods causes a spatially localized viscosity to be felt throughout the computational domain and the locally applied viscosity degrades the accuracy of the entire solution, not just around discontinuities. Additionally, conservative smoothing had difficulty controlling oscillations located close to boundaries. The approach used here will allow the problem to be solved with spectral methods without adding any artificial viscosity.

The superiority of Spectral Methods for the solution of partial differential equations for problems whose solutions possess a certain amount of inherent regularity has been well established. But until recently, spectral methods seemed an inappropriate choice for the solution of hyperbolic problems whose solution may develop sharp discontinuities. By viewing the Gibbs-Wilbraham

oscillations not as noise, but as valuable information from which the physically 
correct solution can be obtained, spectral accuracy can be recovered.

This paper is organized as follows: In Section 2 the Chebyshev pseudospectral method is briefly reviewed. Section 3 summarizes methods developed in [7], [8] and [9] to locate discontinuities, or edges, in the numerical approximations and specializes the method to the Chebyshev pseudospectral approximations. Edge locations will need to be known in order to apply the postprocessing method. Section 4 describes the Gegenbauer reconstruction procedure (GRP) for non-periodic functions developed in [10] and [11]. Numerical results are presented in section 5 .

\section{Chebyshev Collocation Methods}

The standard collocation points for a Chebyshev Collocation (Pseudospectral) method are usually defined by

$$
x_{j}=-\cos \left(\frac{\pi j}{N}\right), \quad j=0,1, \ldots, N .
$$

These points are extrema of the $N^{\text {th }}$ order Chebyshev polynomial,

$$
T_{k}(x)=\cos (k \arccos (x))
$$

The points are often labelled the Chebyshev-Gauss-Lobatto (CGL) points, a name which alludes to the points role in certain quadrature formulas. The CGL points cluster quadratically around the endpoints and are less densely distributed in the interior of the domain.

The Chebyshev Collocation method is based on assuming that an unknown PDE solution, $u$, can be represented by a global, interpolating, Chebyshev partial sum,

$$
u_{N}(x)=\sum_{n=0}^{N} a_{n} T_{n}(x)
$$

The discrete Chebyshev coefficients, $a_{n}$, are defined by

$$
a_{n}=\frac{2}{N} \frac{1}{c_{n}} \sum_{n=0}^{N} \frac{u\left(x_{j}\right) T_{n}\left(x_{j}\right)}{c_{j}} \quad \text { where } \quad c_{j}=\left\{\begin{array}{l}
2 \text { when } j=0, N \\
1 \text { otherwise }
\end{array}\right.
$$


Derivatives of $u$ at the collocation points are approximated by the derivative of the interpolating polynomial evaluated at the collocation points. The first derivative, for example, is defined by,

$$
\frac{d u}{d x}=\sum_{n=0}^{N} a_{n}^{(1)} T_{n}(x) .
$$

Since $a_{N+1}^{(1)}=0$ and $a_{N}^{(1)}=0$, the non-zero derivative coefficients can be computed in decreasing order by the recurrence relation:

$$
c_{n} a_{n}^{(1)}=a_{n+2}^{(1)}+2(n+1) a_{n+1}, \quad n=N-1, \ldots, 1,0 .
$$

The transform pair given by equations (5) or (7) and (6) can be efficiently computed by a fast cosine transform. Equivalently, the interpolating polynomial and its derivatives can be computed in physical space using matrix multiplication. Special properties of the Chebyshev basis allow for differentiation via parity matrix multiplication [12] (even-odd decomposition [13]), which can be performed by using slightly more than half as many floating point operations as standard matrix multiplication.

More detailed information may be found in the standard references [14-19].

After the spectral evaluation of spatial derivatives, the system of ordinary differential equations

$$
\frac{d \mathbf{u}}{d t}=F(\mathbf{u}, t)
$$

results, where $\mathbf{u}$ is the vector containing the unknown PDE solution at the collocation points. The system is typically integrated by a second, third, or fourth order explicit Runge-Kutta method to advance the solution in time.

A coordinate transformation may be necessary either to map a computational interval to $[a, b]$ from the interval $[-1,1]$ or to redistribute the collocation points within an interval for the purpose of giving high resolution to regions of very rapid change. Perhaps, the most popular map used to redistribute the CGL points (3) is the Kosloff/Tal-Ezer map [20]

$$
x=g(\xi, \gamma)=\frac{\arcsin (\gamma \xi)}{\arcsin (\gamma)} \text {. }
$$

If $\xi$ denotes the original variable and $x=g(\xi)$ the new variable, then differentiation of a function $u(x)$ is accomplished by making use of the chain 
rule,

$$
\frac{d u}{d x}=\frac{d \xi}{d x} \frac{d u}{d \xi}=\frac{1}{g^{\prime}(\xi)} \frac{d u}{d \xi},
$$

after a change of variable has been made.

\section{Edge Detection}

The Gegenbauer Reconstruction Procedure (GRP) recovers spectral accuracy up to the discontinuity points in each smooth subinterval of a piecewise analytic function. Thus, the GRP needs the exact location of discontinuities, or edges, in the function. The method used to find the edges originated in [8] for periodic and non-periodic functions. The method is specialized to approximations of functions by Chebyshev methods and is summarized below.

Denote the location of discontinuities as $\alpha_{j}$. Let

$$
[f](x):=f\left(x^{+}\right)-f\left(x^{-}\right)
$$

denote a local jump in the function and define

$$
u e(x)=\frac{\pi \sqrt{1-x^{2}}}{N} \sum_{k=0}^{N} a_{k} \frac{d}{d x} T_{k}(x)
$$

where

$$
\frac{d}{d x} T_{k}(x)=\frac{k \sin (k \arccos (x))}{\sqrt{1-x^{2}}} .
$$

Essentially, we are looking at the derivative of the spectral projection of the numerical solution to determine the location of the discontinuities. The series $u e(x)$ has the convergence properties

$$
u e(x) \rightarrow\left\{\begin{array}{l}
O\left(\frac{1}{N}\right) \text { when } x \neq \alpha_{j} \\
{[f]\left(\alpha_{j}\right) \text { when } x=\alpha_{j}}
\end{array}\right.
$$

The series converges to both the height and direction of the jump at the location of a discontinuity. However, for the GRP, we only need the magnitude

of the jumps. While a graphical examination of the series $u e(x)$ verifies that 
the series does have the desired convergence properties, an additional step is needed to numerically pinpoint the location of the discontinuities. For that purpose, make a non-linear enhancement to the edge series as

$$
u n(x)=N^{\frac{Q}{2}}[u e(x)]^{Q}
$$

The values, un $(x)$, will serve to amplify the separation of scales which has taken place in (11). The series has the convergence properties

$$
u n(x) \rightarrow\left\{\begin{array}{l}
O\left(N^{\frac{-Q}{2}}\right) \quad \text { when } x \neq \alpha_{j} \\
N^{\frac{Q}{2}}\left[[f]\left(\alpha_{j}\right)\right]^{Q} \text { when } x=\alpha_{j}
\end{array}\right.
$$

By choosing $Q>1$ we enhance the separation between the $O\left(\left[\frac{1}{N}\right]^{\frac{Q}{2}}\right)$ points of smoothness and the $O\left(N^{\frac{Q}{2}}\right)$ points of discontinuity. The parameter $J$, whose value will be problem dependent, is a critical threshold value. Finally, redefine $u e(x)$ as

$$
u e(x)= \begin{cases}u e(x) & \text { if } u n(x)>J \\ 0 & \text { otherwise. }\end{cases}
$$

With $Q$ large enough, one ends up with an edge detector $u e(x)=0$ at all $x$ except at the discontinuities $x=\alpha_{j}$. Only those edges with amplitude larger than $J^{1 / Q} \sqrt{1 / N}$ will be detected.

Often the series $u e$ is slow to converge in the area of a discontinuity and the nonlinear enhancement has a difficulty pinpointing the exact location of the edge. If an additional parameter, $\eta$, is added to the procedure this problem can be overcome in a simple manner. The parameter specifies that only one edge may be located in the interval $(x[i-\eta], x[i+\eta]), i=0, \ldots, N$, with appropriate one sided intervals being considered near boundaries. The correct edge will be the maximum of $u e$ in this subinterval. The value of $\eta$ is problem dependent and is best chosen after the edge detection procedure has been applied once.

The edge detection parameters $J, Q$, and $\eta$, are all problem dependent. Various combinations of the parameters may be used to successfully locate edges represented by jumps of magnitude in a certain range. 


\section{Gegenbauer Postprocessing}

The truncation error decays exponentially as $N$ increases when spectral methods are used to approximate smooth functions. However, the situation changes when the function is discontinuous as the spectral approximation no longer converges in the maximum norm. This is known as the Gibbs-Wilbraham phenomenon. Several methods exist for removing or reducing the effects of the Gibbs-Wilbraham phenomenon from spectral approximations. Most however, such as spectral mollification [21], [22], only recover spectral accuracy up to within a neighborhood of each discontinuity. To date, one of the most promising processing method is the Gegenbauer Reconstruction Procedure (GRP). The GRP is capable of recovering spectral accuracy up to and including at the location of discontinuities. It should be noted that the postprocessing method need only be applied at time levels at which a "clean" solution is desired, and not at every time step.

The GRP was developed in [23], [11], [24], [10], and [25] for the purpose of recovering exponential accuracy at all points, including at the discontinuities themselves, from the knowledge of a spectral partial sum of a discontinuous, but piecewise analytic function.

The GRP works by expanding the function in another basis, the Gibbs complementary basis, via knowledge of the known Chebyshev coefficients and the location of discontinuities. The Chebyshev partial sums are projected onto a space spanned by the Gegenbauer polynomials. The associated weight functions increasingly emphasize information away from the discontinuities as the number of included modes grow. The approximation converges exponentially in the new basis even though it only converged very slowly in the original basis due to the Gibbs-Wilbraham phenomenon. The choice of a Gibbs complementary basis is the Ultraspherical or Gegenbauer polynomials, $C_{n}^{\lambda}$. The Gegenbauer polynomials are orthogonal polynomials of order $n$ which satisfy

$$
\int_{-1}^{1}\left(1-x^{2}\right)^{\lambda-1 / 2} C_{k}^{\lambda}(x) C_{n}^{\lambda}(x) d x= \begin{cases}h_{n}^{\lambda} & k=n \\ 0 & k \neq n\end{cases}
$$

where (for $\lambda \geqslant 0)$

$$
h_{n}^{\lambda}=\pi^{\frac{1}{2}} C_{n}^{\lambda}(1) \frac{\Gamma\left(\lambda+\frac{1}{2}\right)}{\Gamma(\lambda)(n+\lambda)}
$$


with

$$
C_{n}^{\lambda}(1)=\frac{\Gamma(n+2 \lambda)}{n ! \Gamma(2 \lambda)} .
$$

Whether the Gegenbauer basis is the optimal choice as the Gibbs complementary basis for the Chebyshev basis remains an open question. It is shown in [11] that the Gegenbauer basis is a Gibbs complementary basis for the Chebyshev basis.

The Gegenbauer expansion of a function $u(x), x \in[-1,1]$ is

$$
u(x)=\sum_{l=0}^{\infty} \widehat{f}_{l}^{\lambda} C_{l}^{\lambda}(x)
$$

where the continuous Gegenbauer coefficients, $\widehat{f}_{l}^{\lambda}$, of $u(x)$ are

$$
\widehat{f}_{l}^{\lambda}=\frac{1}{h_{l}^{\lambda}} \int_{-1}^{1}\left(1-x^{2}\right)^{\lambda-1 / 2} C_{l}^{\lambda}(x) u(x) d x
$$

Since we do not know the function $u(x)$, implementing the GRP requires obtaining an exponentially accurate approximation, $\widehat{g}_{l}^{\lambda}$, to the first $m$ coefficients $\widehat{f}_{l}^{\lambda}$ in the Gegenbauer expansion from the first $N+1$ Chebyshev coefficients of $u(x)$. The approximate Gegenbauer coefficients are defined as the integral

$$
\widehat{g}_{l}^{\lambda}=\frac{1}{h_{l}^{\lambda}} \int_{-1}^{1}\left(1-x^{2}\right)^{\lambda-1 / 2} C_{l}^{\lambda}(x) u_{N}(x) d x
$$

where $u_{N}$ is the Chebyshev partial sum (5). The integral should be evaluated by Gauss-Lobatto quadrature in order to insure sufficient accuracy. The coefficients $\widehat{g}_{l}^{\lambda}$ are now used in the partial Gegenbauer sum to approximate the original function as

$$
u(x) \approx u_{m}^{\lambda}(x)=\sum_{l=0}^{m} \widehat{g}_{l}^{\lambda} C_{l}^{\lambda}(x)
$$

In practice, there will be discontinuities in the interval $[-1,1]$ and the reconstruction must be done on each subinterval $[a, b]$ in which the solution remains smooth. To accomplish the reconstruction on each subinterval, define a local variable for each subinterval as $x(\xi)=\epsilon \xi+\delta$ where $\epsilon=(b-a) / 2$, 
$\delta=(b+a) / 2$ and $\xi_{j}=\cos (\pi j / N)$. The reconstruction in each subinterval is then accomplished by

$$
u_{m}^{\lambda, \epsilon}(\epsilon \xi+\delta)=\sum_{l=0}^{m} \widehat{g}_{\epsilon}^{\lambda}(l) C_{l}^{\lambda}(\xi)
$$

where

$$
\widehat{g}_{\epsilon}^{\lambda}(l)=\frac{1}{h_{l}^{\lambda}} \int_{1}^{-1}\left(1-\xi^{2}\right)^{\lambda-1 / 2} C_{l}^{\lambda}(\xi) u_{N}(\epsilon \xi+\delta) d \xi
$$

Notice that we have used collocation points on the entire interval $[-1,1]$ to build the approximation in $[a, b]$. This is referred to as a global-local approach [25]. The global-local approach seems to be best when postprocessing PDE solutions where $u_{N}$ is obtained from the time evolution. The point values $u\left(x_{i}\right)$ may not be accurate, but the global interpolating polynomial $u_{N}(x)$ is accurate.

In order to show that the GRP yields uniform exponential accuracy for the approximation, it is necessary to select $\lambda$ and $m$ such that $\lambda=m=\beta \epsilon N$, where $\beta<2 e /(27(1+1 / 2 p))$, and $p$ is the distance from $[-1,1]$ to the nearest singularity in the complex plane, in each subinterval where the function being reconstructed is assumed to be analytic [11]. It is not necessary, and usually not advisable, to choose $\lambda=m$. In practice, this condition can be violated and good results can still be obtained.

If the function to be postprocessed consists homogeneous features, the reconstruction parameters can be successfully chosen as $\lambda=k_{\lambda} \epsilon N$ and $m=k_{m} \epsilon N$ for each subinterval where $k_{\lambda}$ and $k_{m}$ are user chosen, globally applied parameters. We refer to this strategy as the global approach.

In the numerical examples the solutions consist of homogeneous features as all solutions are either piecewise constant or piecewise linear. This allows the global approach to choosing the reconstruction parameters to be used which indicates that the problems are particularly well suited to be postprocessed by the GRP. This is because the global approach is currently the easiest and most robust way to apply the method. However, in problems with solutions with varying detail throughout the computational domain, the reconstruction parameters may need to be chosen independently in each subinterval [26]. We refer to this strategy as the local approach. To date there is no known method to choose optimal values of the reconstruction parameters $m$ and $\lambda$. The parameters remain very problem dependent. Work is under way on choosing optimal parameters and results will be reported in a future paper. 


\section{Numerical Examples}

In the numerical examples the postprocessing was done with the Spectral Signal Processing [27] suite. We have chosen two linear examples with exact analytical solutions available. However, the numerical methods apply to nonlinear problems equally as well.

Both examples used the initial conditions $T(x, 0)=0$ and $Q(x, t)=0$ for $x \in$ $[0,1]$. The first example uses boundary conditions of $Q(0, t)=1, Q(1, t)=0$, $T_{t}(0, t)=-Q_{x}(0, t)$, and $T_{x}(1, t)=0$ with the energy generation rate, $S$, set to zero.

In the figure 1 , the temperature solution, $T$, is shown at time $t=0.5$ with $N=33$ CGL grid points (3). The solution was advanced in time with a fourth order Runge-Kutta method and a time step of $\Delta t=0.001$. Strong oscillations are noticeable at the boundary $x=0$, due to the jump in the heat flux, $Q$ ,which is felt by the temperature.

An edge is found to be at $x=0.476$ with the parameters $J=200, Q=4$, and $\eta=2$. This choice of edge detection parameters results in jumps of 0.65 and larger being located. The exact jump is 0.65 in magnitude. By specifying $\eta=2$, the oscillation near $x=0$ is not falsely determined to be a jump in the function. With only 33 grid points, the convergence of the edge series, figure 2 , is not yet readily apparent, however, if the edge detection parameters are chosen appropriately, the correct edge locations will be found.

After the edges have been located, the GRP is applied in each smooth subinterval with by using global parameters chosen as $k_{\lambda}=0.3$ and $k_{m}=0.1$ which results in $m=2$ and $\lambda=4.7$ in subinterval $(0,0.476)$ and $m=2$ and $\lambda=5.2$ in subinterval $(0.476,1)$.

After postprocessing (figure 3) the numerical and exact solutions are virtually identical. The pointwise error between the exact and postprocessed solution is shown in figure 4 where the maximum pointwise error is less than 0.00045 . Only $1 / 30$ of the grid points are necessary to obtain results superior to those obtained in [2] with MacCormack's method. Figure 5 displays the MacCormack's method solution of the first example with $N=1000$ and a small time step. Despite using substantially more grid points the solution is still oscillatory around the steep front due mainly to phase speed errors. Spectral methods give accurate phase speeds for all modes, while second order finite difference methods typically only resolve first few modes accurately [28]. Unlike the spectral solution, there is no known postprocessing technique which is capable of recovering second order accuracy over the entire computational domain of the finite difference approximation. 
The second example uses boundary conditions of $Q(0, t)=0, Q(1, t)=0$, $T_{x}(0, t)=0$, and $T_{x}(1, t)=0$, with the energy generation rate specified as $S(x, t)=\frac{1}{d n}$ if $0 \leq x \leq d n$ and zero otherwise. The energy generation rate, $S$, represents a pulsed energy source released instantaneously at time $t=0$. Such an energy source could model the application of a strong laser pulse at the boundary of an absorbing medium encountered in the annealing of semiconductors.

The temperature solution with $d n=0.05$ is shown at time $t=0.5$ with $N=99$ grid points distributed with the map (9) with $\gamma=0.96$. By taking the map parameter as $\gamma=0.96$, the grid becomes closer to evenly spaced and better resolution is realized in the center of the domain. The solution (figure 6) was advanced in time with a fourth order Runge-Kutta method and a time step of $\Delta t=0.0005$.

Edges, figure 7 , are found to be at $x=0.447$ and $x=0.541$ with the parameters $J=5000, Q=3$, and $\eta=1$. With these choices of the edge detection parameters, only jumps of magnitude greater than 1.72 are found. Other combinations of $J$ and $Q$ could work equally as well.

After the edges have been located, the GRP is applied in each smooth subinterval by using the global parameters $k_{\lambda}=0.2$ and $k_{m}=0.02$. The results are shown in figure 8.

\section{Conclusions}

The Chebyshev collocation method in conjunction with the Gegenbauer reconstruction procedure can produce superior results with significantly fewer grid points than previously applied finite difference and finite element methods to problems of hyperbolic heat transfer. Additionally, the results are achieved without any artificial viscosity being used. The techniques used in this paper can be applied to problems in higher dimensions where the storage savings would be even greater.

\section{References}

[1] G.F. Carey and M. Tsai. Hyperbolic heat transfer with reflection. Numerical Heat Transfer, 5:309-327, 1982.

[2] D.E. Glass, M.N. Ozisik, D.S. McRae, and Brain Vick. On the numerical solution of hyperbolic heat conduction. Numerical Heat Transfer, 8:497-504, 1985. 
[3] D.E. Glass, M.N. Ozisik, and D.S. McRae. Hyperbolic heat conduction with radiation in an absorbing and emitting medium. Numerical Heat Transfer, 12:321-333, 1987.

[4] P.L. Roe. Approximate Riemann solvers, parameter vectors, and difference schemes. Journal of Computational Physics, 43:357-372, 1981.

[5] B. P. Leonard. The ULTIMATE conservative difference scheme applied to unsteady one-dimensional advection. Computer Methods in Applied Mechanics and Engineering, 88:17-74, 1991.

[6] Chris Guenther. Pseudospectral Techniques for Non-Smooth Evolutionary Problems. PhD thesis, West Virginia University, 1998.

[7] Anne Gelb and Eitan Tadmor. Detection of edges in spectral data. Applied and Computational Harmonic Analysis, 7:101-135, 1999.

[8] Anne Gelb and Eitan Tadmor. Detection of edges in spectral data II: Nonlinear enhancement. SIAM Journal of Numerical Analysis, 38:1389-1408, 2000.

[9] Anne Gelb and Eitan Tadmor. Enhanced spectral viscosity approximations for conservation laws. Applied Numerical Mathematics, 33:3-21, 2000.

[10] David Gottlieb and Chi-Wang Shu. On the Gibbs phenomenon IV: Recovering exponential accuracy in a subinterval from a Gegenbauer partial sum of a piecewise analytic function. Mathematics of Computation, 64:1081-1095, 1995.

[11] David Gottlieb and Chi-Wang Shu. On the Gibbs phenomenon and its resolution. SIAM Review, 39(4):644-668, 1997.

[12] John P. Boyd. Chebyshev and Fourier Spectral Methods. Dover Publications, Inc, New York, second edition, 2000.

[13] A. Solomonoff. A fast algorithm for spectral differentiation. Journal of Computational Physics, 98:174-177, 1992.

[14] Claudio Canuto, M. Y. Hussaini, Alfio Quarteroni, and Thomas A. Zang. Spectral Methods for Fluid Dynamics. Springer-Verlag, New York, 1988.

[15] Benjt Fornberg. A Practical Guide to Pseudospectral Methods. Cambridge University Press, New York, 1996.

[16] D. Funaro. Polynomial Approximation of Differential Equations. SpringerVerlag, New York, 1992.

[17] David Gottlieb and Steven A. Orszag. Numerical Analysis of Spectral Methods. SIAM, Philadelphia, PA, 1977.

[18] David Gottlieb, M. Y. Hussaini, and Steven A. Orszag. Theory and application of spectral methods. In R. G. Voigt, D. Gottlieb, and M. Y. Hussaini, editors, Spectral Methods for Partial Differential Equations, pages 1-54. SIAM, Philadelphia, 1984.

[19] L. N. Trefethen. Spectral Methods in Matlab. SIAM, Philadelphia, 2000. 
[20] R. Kosloff and H. Tal-Ezer. A modified Chebyshev pseudospectral method with an $\mathrm{O}(1 / \mathrm{N})$ time step restriction. Journal of Computational Physics, 104:457469, 1993.

[21] D. Gottlieb and Eitan Tadmor. Recovering pointwise values of discontinuous data within spectral accuracy. In Progress in Scientific Computing, number 6 in Proceedings of U.S.-Israel Workshop, 1984, pages 357-375. Birkhauser Boston, 1985.

[22] Eitan Tadmor and Jared Tanner. Adaptive mollifers - high resolution recovery of piecewise smooth data from its spectral information. preprint, March 2001.

[23] David Gottlieb, C.-W. Shu, A. Solomonoff, and H. Vandeven. On the Gibbs phenomenon I: recovering exponential accuracy from the Fourier partial sum of a nonperiodic analytic function. Journal of Computational and Applied Mathematics, 43:81-98, 1992.

[24] David Gottlieb and Chi-Wang Shu. On the Gibbs phenomenon III: Recovering exponential accuracy in a subinterval from a partial sum of a piecewise analytic function. SIAM Journal of Numerical Analysis, 33:280-290, 1996.

[25] David Gottlieb and Chi-Wang Shu. On the Gibbs phenomenon V: Recovering exponential accuracy from collocation point values of a piecewise analytic function. Numerische Mathematik, 71:511-526, 1995.

[26] Scott A. Sarra. Chebyshev Collocation Methods for Conservation Laws with Source Terms with applications to Multiphase Flow. PhD thesis, West Virginia University, 2002.

[27] Scott A. Sarra. Spectral Signal Processing. submitted: ACM Transactions on Mathematical Software, 2002. www.scottsarra.org/signal/signal.html.

[28] B. E. McDonald. Flux-corrected pseudospectral method for scalar hyperbolic conservation laws. Journal of Computational Physics, 82:413-428, 1989. 


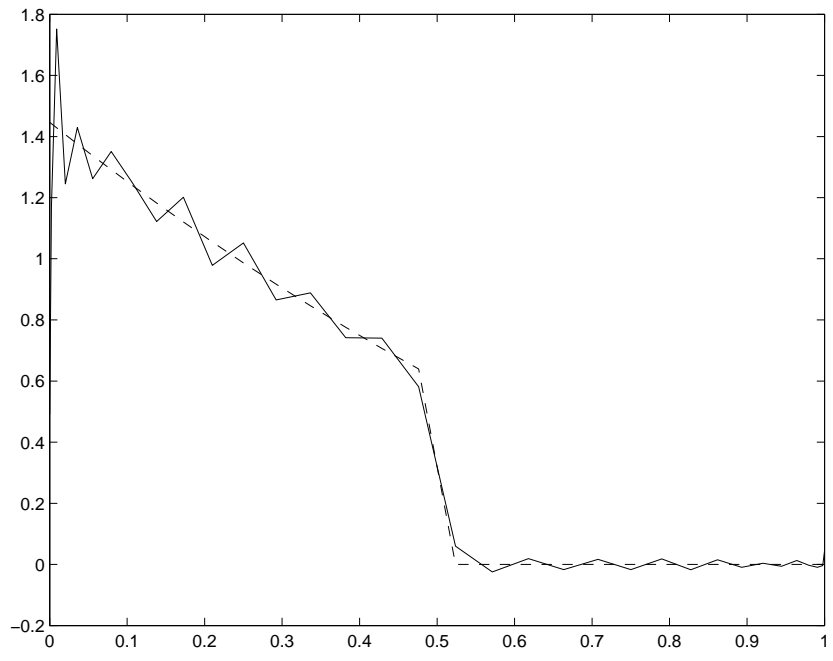

Fig. 1. spectral (solid) vs. exact 


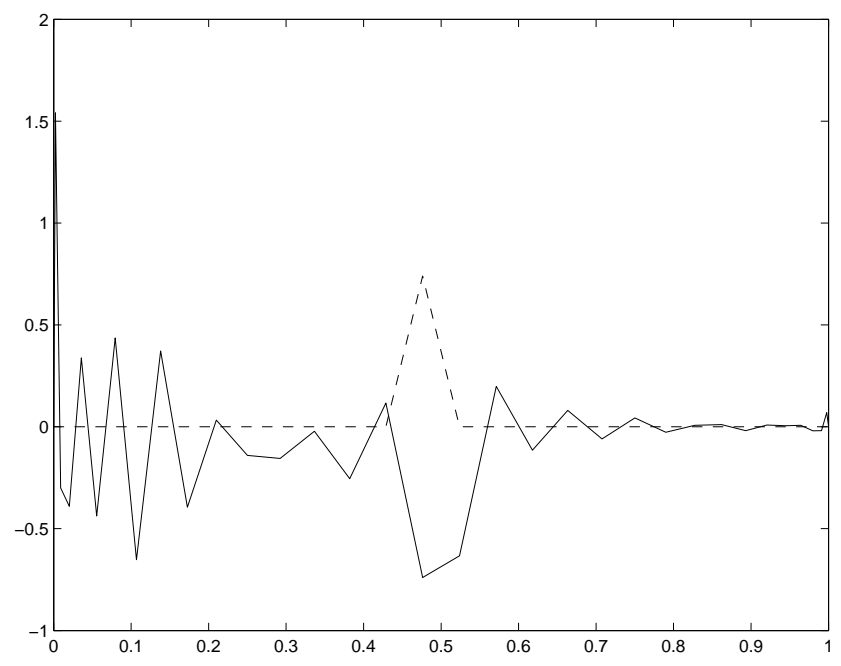

Fig. 2. enhancement and edge series (solid) 


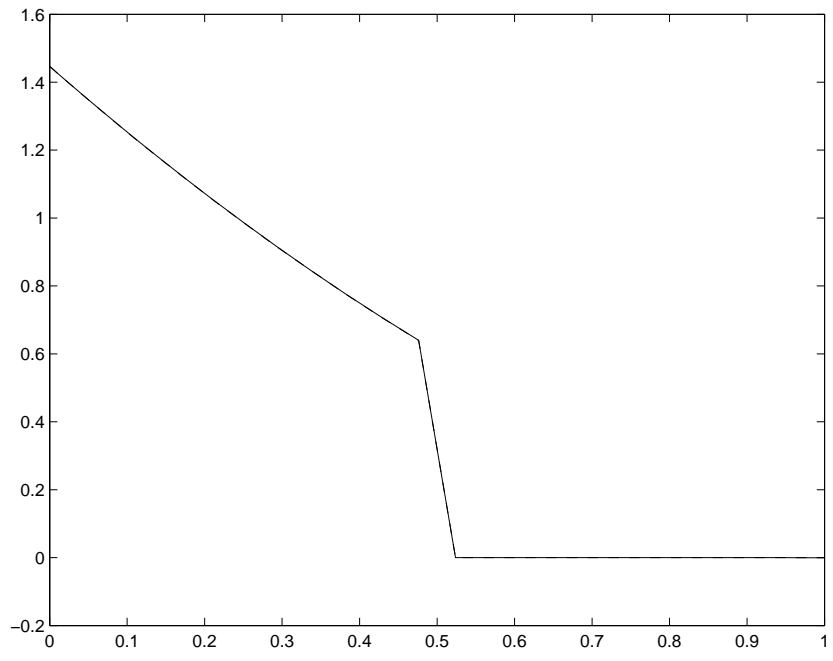

Fig. 3. postprocessed (solid) vs. exact 


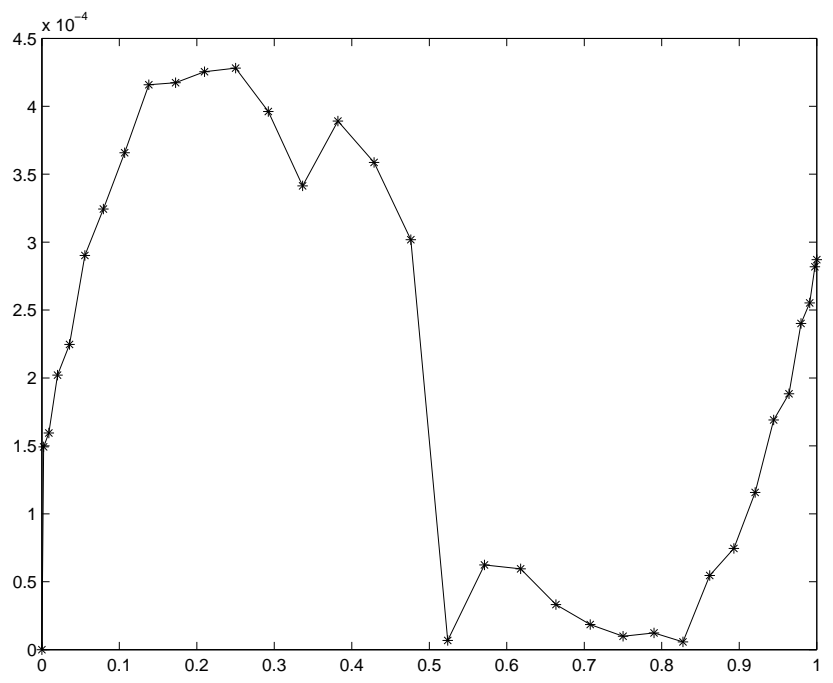

Fig. 4. pointwise error, |postprocessed - exact $\mid$ 


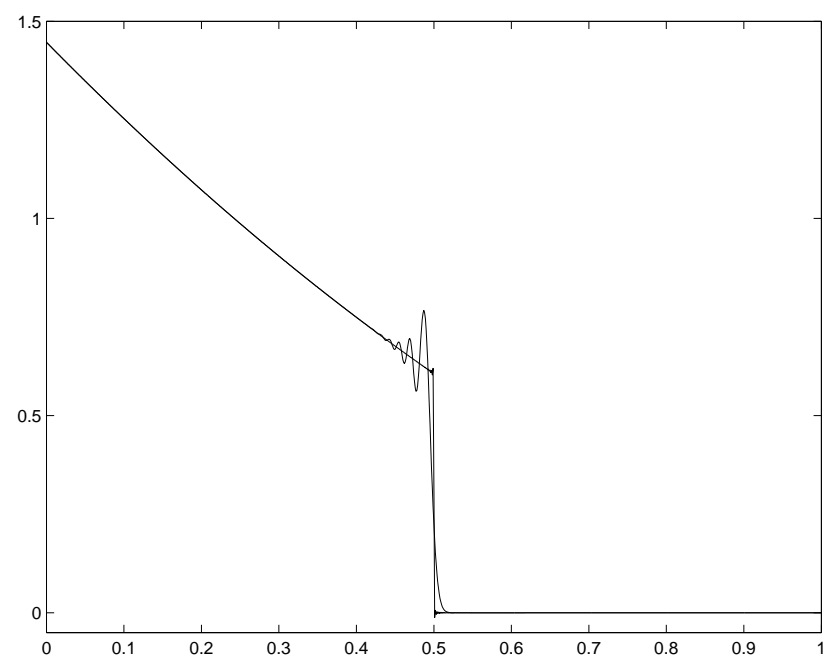

Fig. 5. Problem 1: MacCormack's (solid) vs. exact 


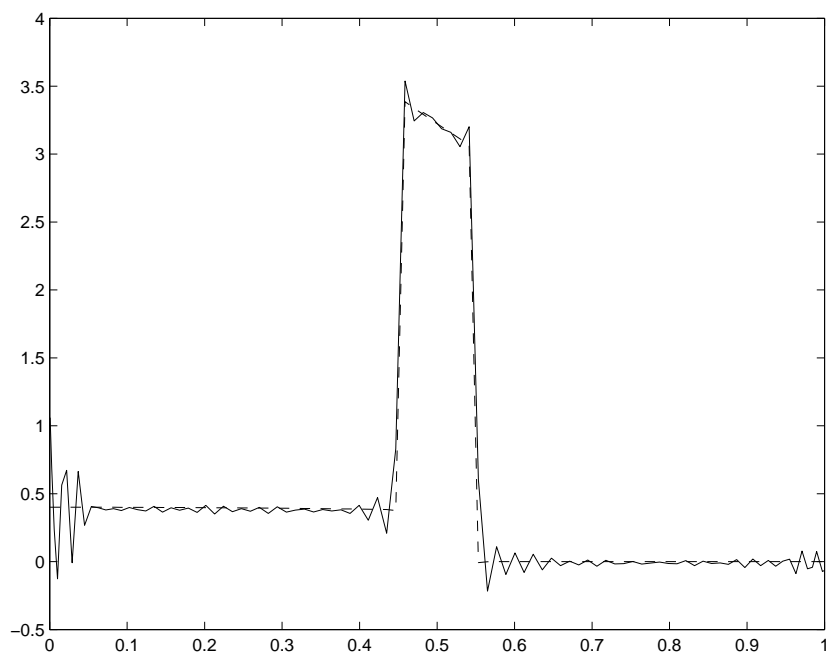

Fig. 6. spectral (solid) and exact 




Fig. 7. enhancement (solid) and edge series 


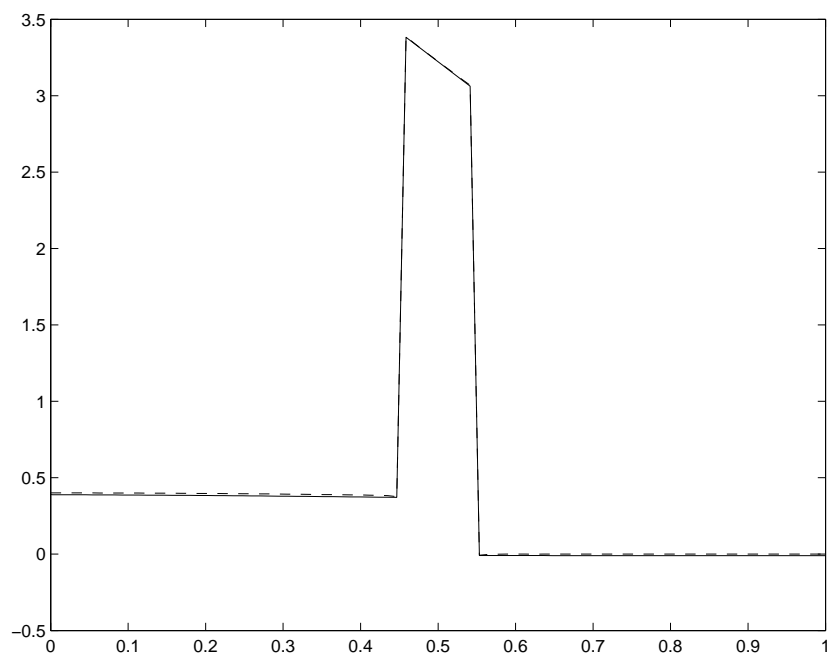

Fig. 8. postprocessed (solid) vs. exact 